\title{
ENFERMERAS ASISTENCIALES y LA PRÁCTICA DE INVESTIGACIONES: ESTUDIO DE UNA REALIDAD HOSPITALARIA *
}

\author{
Célia Alves Rozendo ** \\ Neusa Collet *** \\ Maria Cícera dos S. de Albuquerque ****
}

El presente trabajo busca demostrar la situación de las enfermeras de una determinada institución hospitalaria en relación a la investigación en enfermería, enfocando la atención en la investigación anterior y actual, en los elementos limitantes y propiciadores para la elaboración del trabajo científico y en el significado que tiene la investigación para la enfermería, en la Opinión de las participantes del estudio. En la colecta de datos fueron utilizados como instrumentos: cuestionario y entrevista semiestructurada.

UNITERMOS: investigación en enfermería, enfermeras asistenciales

\footnotetext{
*Trabajo presentado en IV COLOQUIO PANAMERICANO DE INVESTIGACIÓN EN ENFERMERÍA, ocurrido en el periodo de 23-25 de noviembre de 1994, en Concepción/Chile.

**Enfermera, alumna de maestría del Programa de Postgraduación en Enfermería (Fundamental de la Escuela de Enfermería de Ribeirão Preto de la Universidad de São Paulo, docente del Departamento de Enfermería de la Universidad Federal de Alagoas, Maceió Brasil.

*** Enfermera, alumna de maestría del Programa de Postgraduación en Enfermería Fundamental de la Escuela de Enfermería de Ribeirão Preto de la Universidad de São Paulo, docente del Departamento de Enfermería de la Universidad del Oeste de Paraná, Cascabel Brasil.

**** Enfermera, magíster en educación, docente del Departamento de Enfermería de la Universidad Federal de Alagoas, Maceió - Brasil.
} 


\section{INTRODUCCIÓN}

Las tentativas de explicación de la realidad acompañan al hombre desde tiempos remotos, o sea, desde el momento indefinido en que apareció la percepción de la necesidad de conocerse a sí mismo y al mundo en que habita, buscando en la naturaleza, de inicio, los medios para satisfacer tales necesidades.

La inagotable curiosidad y necesidad humanas de conocer, resisten al tiempo y a las adversidades, determinando y siendo determinadas muchas veces por éstos. Por tanto, hombre, tiempo y necesidades son elementos inseparables dentro del proceso del conocimiento, cuyo intervalo va desde el pensamiento mágico hasta el llamado conocimiento científico. Este último fue elaborado, a su vez, a partir del siglo pasado y se utiliza desde varias vertientes para aprender y explicar la realidad.

De esta manera, el conocimiento científico va siendo acumulado, renovado y transformado a lo largo de la existencia humana en busca de respuestas a sus inquietudes y satisfacción de sus necesidades. Esto permite suponer que el conocimiento científico atiende a las demandas humanas de naturaleza e intereses más diversos, así como a la transformación de la realidad.

Incorporando este raciocinio a la enfermería, creemos que el conocimiento científico deba constituir el hilo conductor de los principios que guíen la profesión.

Nuestra intención no es hacer apología del conocimiento científico, tampoco de la investigación como recurso por medio del cual se produce el conocimiento. Sin embargo, consideramos que el entendimiento acerca de éstos, por parte de los enfermeros, es fundamental para el conocimiento de la enfermería como profesión, sin, no obstante, perder de vista otros determinantes.

Coincidimos con NOGUEIRA ${ }^{5}$, cuando expresa que "la investigación en enfermería es importante porque existe necesidad - como hubo en otras profesiones de que ella se autodefina como profesión, y quizás, como desean algunos, hasta como ciencia, con un cuerpo propio del conocimiento". Por tanto, es menester un esfuerzo colectivo de los profesionales en este sentido.

En este trabajo pretendemos enfocar una parte de estos profesionales, a saber, los enfermeros asistenciales. Todavía, antes de hacer este recorte, juzgamos necesario realizar un relato sucinto de la evolución histórica de la investigación en enfermería en Brasil.

Aunque con un considerable crecimiento en las últimas tres décadas, la investigación en enfermería en Brasil aun es incipiente, especialmente cuando se refiere a la producción científica de los enfermeros asistenciales .Hasta la década del 70 el número de trabajos producidos fue bastante reducido, con la primera investigación importante realizada por la ABEn (Associação Brasileira de Enfermagem) en 1957, titulada "Levantamiento de Recursos Humanos y Necesidades de Enfermería en el Brasil". A partir de aquí fueron desarrollados algunos trabajos de tesis de doctorado, entre los cuales destacamos dos de los pioneros: "A Enfermagem Moderna como Categoria Profissional: Obstáculos para sua Expansão na Sociedade Brasileira", (1963), y “A Enfermagem como Profissão” (1973).

La literatura consultada revela que la investigación en la enfermería brasilera tomó impulso a partir de la década del 70 , momento en que se criaron los 
primeros cursos de postgrado a nivel de maestría, como resultado de la exigencia en la elaboración de investigaciones para la obtención de tal grado. De esta manera, la producción científica en esta área guarda relación directa con la postgrado, situación que persiste hasta el presente, según refiere NOGUEIRA ${ }^{5}$ : "en la mayoría de las veces, la producción científica de la enfermería brasilera ha estado, hasta a hora, vinculada a los postgrado, o sé a, a la producción de investigaciones para la obtención de títulos".

En 1971, un acontecimiento que realmente causó gran impacto en el desarrollo de la investigación en enfermería, fue la creación del "Centro de Pesquisas en Enfermagem" (CEPEn), durante una asamblea de delegados de la ABEn. El CEPEn fue oficialmente incorporado a la ABEn como uno de sus órganos en 1976, a través de la reforma de sus estatutos, según afirma MACHADO ${ }^{3}$. Relativo a esto, ROZENDO \& ALBUQUERQUE" refieren que "desde entonces el CEPEn ha representado un importante canal de comunicación entre los investigadores y ha servido como vehículo de diseminación del conocimiento producido", especialmente mediante los seminarios nacionales de investigación en enfermería.

Los profesionales de esta área han mostrado preocupación e interés en el asunto, lo cual está siendo punto de discusiones en reuniones científicas y de investigaciones. MENDES et $\mathrm{al}^{4}$, comentan que los esfuerzos desplegados y el análisis crítico que está siendo efectuado han contribuido a la mejoría de la calidad de las investigaciones. No obstante, la investigación científica así como la propia enfermería aun enfrentan muchas dificultades y limitaciones, generando un cuadro de aspectos peculiares descritos a continuación.

El primero de éstos es el nexo de la investigación con los postgrados. Fuera de este ámbito, su práctica es escasa, lo que equivale a decir que la mayoría de los trabajos son realizados por enfermeros docentes, una vez que éstos ocupan la mayor parte de las plazas en los cursos existentes en Brasil, lo cual implica la predominancia de trabajos de autoría individual. Según $\mathrm{MACHADO}^{3}$, este hecho revela la ausencia de grupos de investigadores, factor esencial en la investigación moderna.

El segundo aspecto se refiere a la falta de establecimiento de prioridades y líneas de investigación, lo cual está íntimamente relacionado con el aspecto anterior. Tales prioridades podrían ser establecidas a medida que los investigadores evaluaran la aplicabilidad de sus hallazgos y a partir de ahí, la necesidad de nuevas investigaciones. Además de estas limitaciones, podemos citar otras: el reducido número de centros de investigaciones; la sobrecarga de actividades que en general recae sobre los enfermeros; las limitaciones de los enfermeros relativo a metodologías de investigación; un deficiente sistema de intercambio entre enfermeros y escasa divulgación de las investigaciones realizadas ${ }^{5}$.

Finalmente, destacamos la falta de interacción entre investigadores y consumidores en potencial de investigación-los enfermeros asistenciales. Para MENDES et $\mathrm{al}^{4}$, "la interrelación del investigador con los consumidores constituye el punto neurálgico de la cuestión, porque son ellos (en este caso los enfermeros de servicio) los que pondrán los resultados de investigación en práctica". Estas autoras consideran que la interrelación entre los enfermeros de servicio es necesaria para la retroalimentación del saber producido. Pensamos que esta intercomunicación no se debería limitar al consumo de investigaciones, resultando indispensable su realización 
por los enfermeros, a partir de sus propias vivencias profesionales y de las necesidades que de ellas se desprenden.

En este trabajo, ya referimos que el número de investigaciones elaboradas por enfermeros asistenciales es pequeño y son pocos los que frecuentan los cursos de postgraduación. Los obstáculos referidos por éstos son muchos, como demuestran CASTELLANOS \& SALUM ${ }^{1}$ : acumulo de actividades, poca disponibilidad de tiempo, falta de recursos materiales y humanos, poca preparación y falta de incentivo y apoyo institucionales, entre otros.

El deseo de conocer más sobre esta realidad, así como el interés y preocupación por el tema abordado, en particular las cuestiones relativas a la participación del enfermero asistencial, nos llevaron a la realización del presente trabajo. El objetivo es retratar la situación de los enfermeros de una determinada institución hospitalaria en relación a la investigación en enfermería, con énfasis:

- en la participación en investigación anterior y actual;

- en los elementos limitantes y propiciadores para la elaboración del trabajo científico;

- en el significado que tiene la investigación para la enfermería de acuerdo con la opinión de las investigadas;

- en el deseo y capacidad para realizar investigaciones.

\section{METODOLOGÍA}

La población estuvo integrada por los enfermeros de la Santa Casa de Misericordia de la ciudad de Piracicaba, en el Estado de São Paulo-Brasil, con un total de veintidós participantes, de los veinticuatro que constituían la totalidad del referido local de la investigación. Los datos fueron colectados en el mes de mayo de 1994, después de contacto anterior con la dirección del servicio de enfermería.

El medio usado para colectar los datos fue un cuestionario (encuesta) compuesto por diez preguntas, con una abierta y nueve cerradas, las cuales muestran dos alternativas y solicitud de justificación de las respuestas. Las informaciones referentes a la institución fueron obtenidas a partir de entrevista realizada con la dirección del Servicio de Enfermería del hospital en estudio.

Después de colectados, los datos fueron agrupados tomando en cuenta los objetivos propuestos, distribuidos en tablas según las siguientes variables: edad, estado civil, tiempo y nivel de formación (Tabla 1), carga horaria de trabajo, vínculos laborales, realización de investigaciones previas (Tabla 2), acceso a la literatura, hábito de lectura científica y realización de investigaciones actuales (Tabla 3), de manera a establecer relaciones entre si y representados como frecuencias absolutas, cuyo análisis fue efectuado a la luz de la literatura consultada. Los datos de la octava pregunta incluida en el cuestionario no fueron analizados, dado que los resultados obtenidos fueron muy inconsistentes. Se optó por garantizar las informaciones por medio de la entrevista semiestructurada con la dirección del Servicio de Enfermería.

La novena pregunta fue analizada parcialmente debido a la ambigüedad de las respuestas. 


\section{PRESENTACIÓN Y ANÁLISIS DE LOS RESULTADOS}

No todos los resultados encontrados aparecen en las tablas, especialmente aquellos provenientes de la pregunta abierta, en función de la diversidad de respuestas, dificultando así, su tabulación. Además, consideramos dispensable exponer todos ellos en las tablas y optamos por realizar sus análisis por separado, pero haciendo relaciones con las otras cuestiones propuestas en el trabajo y con el contexto de manera general.

TABLA 1 - CARACTERIZACIÓN DE LOS INDIVIDUOS INVESTIGADOS DE ACUERDO CON LA EDAD, ESTADO CIVIL, TIEMPO Y NIVEL DE FORMACIÓN

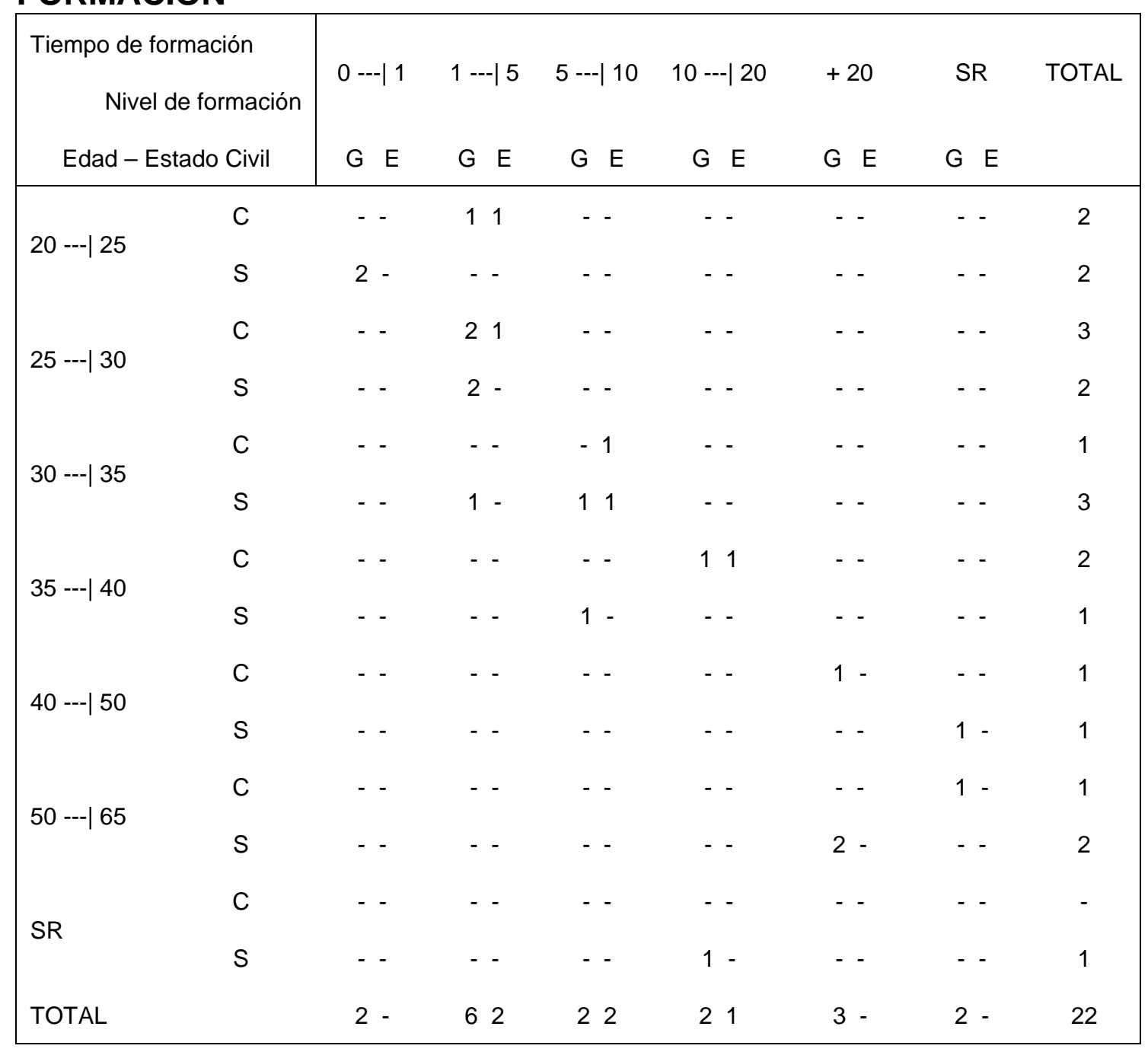

Fuente: cuestionario usado en la investigación

Convenciones: G - Graduación; E - Especialización; S - Soltero; C - Casado; SR - Sin Respuesta 
Como muestra la Tabla 1, la mayoría de los encuestados, todas mujeres, estaban entre 20 e 40 anos de edad (16), solteras (12), formadas en los últimos diez anos (14) y sin curso de postgraduación (17). Las cinco enfermeras postgraduadas son especialistas y tienen menos de 40 anos. De estas últimas, cuatro son casadas y con tiempo de formación entre uno y diez anos.

El resultado es, por tanto, un número bastante reducido de enfermeras con postgrado y, en este caso, a nivel de especialización. Considerando que, en la realidad brasilera, frecuentemente el inicio en la investigación tiene lugar a través de los cursos de maestría, puede inferirse que este grupo de enfermeras no se encuentra, desde el punto de vista de titulación, en condiciones favorables para la elaboración de investigaciones científicas. Este cuadro no resulta restringido a la población estudiada, como muestran ROZENDO \& ALBUQUERQUE ${ }^{7}$.

\section{TABLA 2 - DISTRIBUCIÓN DE LOS ENCUESTADOS EN RELACIÓN CON LA CARGA MORARÍA DE TRABAJO EN LA INSTITUCIÓN INVESTIGADA, OTRO VÍNCULO LABORAL Y RELACIÓN DE INVESTIGACIONES PREVIAS.}

\begin{tabular}{|c|c|c|c|c|c|c|}
\hline \multirow{2}{*}{\multicolumn{2}{|c|}{$\begin{array}{l}\text { Tiempo de formación } \\
\qquad \text { Nivel de formación }\end{array}$}} & \multirow[b]{2}{*}{36} & \multirow[b]{2}{*}{40} & \multirow[b]{2}{*}{44} & \multirow[b]{2}{*}{+44} & \multirow{3}{*}{ TOTAL } \\
\hline & & & & & & \\
\hline & Civil & $S N$ & $S N$ & $S N$ & $S \mathrm{~N}$ & \\
\hline \multirow{2}{*}{$S$} & $\mathrm{~S}$ & - & -1 & - & - & 1 \\
\hline & $N$ & - & - & - & - & - \\
\hline \multirow{2}{*}{$\mathrm{N}$} & $S$ & -1 & -2 & -1 & -1 & 5 \\
\hline & $\mathrm{N}$ & $1-$ & 25 & -2 & -3 & 13 \\
\hline SR & & -2 & - & - & -1 & 3 \\
\hline TOTAL & & 13 & 28 & -3 & -5 & 22 \\
\hline
\end{tabular}

Fuente: cuestionario usado en la investigación

Convenciones: S - Sí; N - No; SR - Sin Respuesta 
En la introducción del presente trabajo expusimos que la tradición de investigación entre enfermeros asistenciales es escasa y que la producción científica en esta área es predominantemente realizada por docentes. Esta afirmación resulta corroborada por los datos de la Tabla 2, según la cual, dieciocho de las veintidós enfermeras encuestadas no desarrollan investigación actualmente, tres no respondieron el cuestionario y sólo una está ligada a esta área actualmente. Los datos que disponemos, sin embargo, no permiten identificar el contenido y naturaleza del trabajo desarrollado por esta enfermera.

Comprobamos que, de los pocos trabajos realizados por las encuestadas, tres estaban vinculados con la graduación o postgraduación, o sea, posiblemente fueron elaborados por exigencia académica. Dos tuvieron relación con la actividad profesional, lo cual, si bien un número reducido, puede significar la existencia de interés no manifiesto entre los profesionales estudiados. Tal interés, creadas las condiciones necesarias, podría transformarse en una práctica concreta.

La mayoría de estas enfermeras trabaja, al menos, 40 horas semanales (18) y no tienen otro vinculo laboral (19). Aquellas que laboran 36 horas se desempeñan en lo que erróneamente se ha dado en llamar supervisión, función en la cual la enfermera responde por las actividades de enfermería de todo el hospital en el momento en que no están presentes enfermeras en todas las unidades, en el caso de la institución estudiada, a partir de las 16 horas.

Las enfermeras encuestadas consideran que la falta de tiempo, de asesoramiento y motivación, dificultades de financiamiento, falta de preparación, ausencia de incentivo institucional e inexperiencia constituyen las causas principales que impiden su participación en la investigación.

CASTELLANOS \& SALUM ${ }^{1}$, publicaron hallazgos semejantes en una investigación efectuada con enfermeros asistenciales, los cuales, "en su trabajo cotidiano, identifican que la investigación es dificultada: por el cúmulo de actividades de rutina, por la escasa disponibilidad de tiempo (el trabajo administrativo unido al asistencial exige mucho del enfermero), por la falta de recursos materiales y (ya que las instituciones no asumen la investigación como un objetivo) humanos y dado el pequeño número de profesionales dispuestos a investigar-la investigación es un rubro de gastos, existe falta de apoyo institucional, lo cual conduce al acomodamiento".

Consideramos que, en el contexto aquí reflejado, el "acomodamiento" es algo hasta cierto punto comprensible. Sabemos que, en la mayoría de las instituciones hospitalarias del país, los enfermeros representan una parte reducida de la fuerza de trabajo en enfermería. Su trabajo cotidiano es recargado de actividades que, muchas veces, no resultan de su competencia. Se da énfasis a las tareas de carácter administrativo que frecuentemente no tienen una relación directa en la asistencia y que pueden ser realizadas por otros trabajadores. Aún de acuerdo con las autoras arriba citadas, "los profesionales de enfermería desconocen el proceso de investigación, pues no han sido preparados para actuar como investigadores: no se sitúa, durante al proceso de aprendizaje, la importancia de la aplicación de la 
investigación de manera concreta: existe poco incentivo, dificultad y falta de experiencia teórico-práctica en relación a la investigación en los cursos de graduación".

La realización de cursos de actualización también es dificultada, ya que la frecuencia de éstos es reducida, dado que se trata de una población de enfermeras que trabajan en una institución hospitalaria de una ciudad del interior, donde no existen cursos superiores de enfermería.

Además de las limitaciones antes mencionadas, la literatura consultada revela asimismo que, aún cuando los enfermeros asistenciales "hayan tenido acceso a cursos específicos, no encuentran condiciones y seguridad para iniciar o dar curso a investigaciones, dada las limitaciones para encontrar bibliografía adecuada (...). Consideran además que dificultades tales como: la falta de refección sobre el trabajo diario; la remuneración inadecuada; las condiciones de trabajo, a veces pésimas; el trabajo aislado de un enfermero solo, condicionan desestímulo para realizar cualquier cosa que salga fuera de la rutina cotidiana"1.

CASTRO et al., citados por LOPES ${ }^{2}$, afirman que los enfermeros confrontan dificultades para evaluar la aplicabilidad de las investigaciones, así como para conocer sus resultados. Para estas autoras, tal situación es resultado de la falta de hábito de lectura, de inseguridad para apreciar la calidad de los resultados y de la precaria situación en el intercambio de informaciones en la enfermería.

\section{TABLA 3 - DISTRIBUCIÓN DE LOS ENCUESTADOS DE ACUERDO CON LA REALIZACIÓN DE LA INVESTIGACIÓN ACTUAL, RELACIONADA CON EL ACCESO A LA LITERATURA Y EL HÁBITO DE LECTURA CIENTÍFICA.}

\begin{tabular}{|c|cccc|}
\hline $\begin{array}{c}\text { Aceso a la literatura } \\
\text { Hábito de lectura }\end{array}$ & S & N & SR & TOTAL \\
Edad - Estado Civil & S N SR & S N SR & S N & \\
\hline S & $-\cdots$ & $1-$ & $-\cdots$ & 1 \\
N & $51-$ & $64-$ & --2 & 18 \\
SR & $-\cdots-1-1$ & 3 \\
TOTAL & $51-$ & $84-$ & $1-3$ & 22 \\
\hline
\end{tabular}

Fuente: cuestionario usado en la investigación

Convenciones: S -Sí; N -No; SR - Sin Respuesta 
Partiendo de estos elementos, el análisis de la Tabla 3 muestra que del total de las enfermeras encuestadas, doce refieren no tener acceso a literatura científica, en tanto dieciocho no desarrollan investigación actualmente. Entre las que no tienen acceso, ocho cultivan el hábito de leer materiales de naturaleza científica, seis refieren tener acceso a la literatura, resultando que cinco de ellas dicen que leen habitualmente y una no respondió si tiene o no acceso a la literatura, sin embargo, afirma tener el hábito de leer literatura científica. Por tanto, son catorce las enfermeras que afirman leer materiales de naturaleza científica.

Los datos obtenidos no ofrecen elementos que posibiliten conocer el tipo de literatura accesible, o sea, si son libros, publicaciones periódicas o ambos. No obstante, pudimos constatar que apenas tres de las personas encuestadas suscriben alguna revista, justificando que lo hacen por necesidad de actualización. La mayoría (14) afirma que no está suscrito a ninguna revista, siendo el motivo expuesto con mayor frecuencia, la falta de recursos financieros. Esto nos lleva a suponer que la literatura más accesible a este grupo son los libros académicos.

La entrevista realizada con la Dirección del Servicio de Enfermería posibilitó conocer que la institución no mantiene suscripciones de revistas actualmente, aunque dispone de algunos números recibidos como donativos; no posee biblioteca facilitar y estimular la participación de las enfermeras en eventos científicos; el equipo de enfermería es compuesto por 24 enfermeras (dos estaban separadas del trabajo en el momento de la realización de esta investigación), 15 técnicos, 68 auxiliares e 243 ayudantes de enfermería y existen 380 camas. Vale destacar que la mayoría de las enfermeras participantes del estudio reciben en tomo de ocho salarios mínimos, unos 512 dólares.

Vemos, entonces, que el cuadro presentado por la institución, de manera general, no favorece la realización de trabajos científicos por las enfermeras, aunque exista interés expreso de aquella en tal sentido. Consideramos que la sobrecarga de trabajo, los bajos salarios, la falta de calificación de los recursos humanos y la ausencia de incentivo institucional, constituyen algunos de los factores limitantes para la inserción de estas enfermeras en la esfera de la investigación científica. Estas condiciones de trabajo concuerdan en gran medida con las referidas en la literatura consultada.

A pesar de este cuadro poco estimulante, coincidimos con RIBEIRO': "para la sobre vivencia de la profesión la investigación sistemática es necesaria para ampliar la base de conocimientos para su práctica más efectiva. La investigación constituye el apoyo de la práctica, sin la cual ésta se toma rutina. $Y$ rutina los ayudantes son capaces de ejecutar transformándose, la enfermera en este caso, en simple instrumento de control, con pérdida de sus características profesionales".

Los datos obtenidos revelan, además, que la mayoría de las enfermeras incluidas en el estudio comprenden la importancia de la investigación para la enfermería y sienten deseos de participar en este tipo de actividad, aun cuando no todas se consideran capacitadas para tal. 
En la pregunta abierta del instrumento utilizado para la colecta de datos, procuramos aprehender el significado que la investigación tiene para la enfermería según la visión de las encuestadas, de las cuales tres omitieron sus opiniones. Para la mayor parte, la investigación asume gran importancia para la profesión, como muestran las siguientes opiniones: "torna la enfermería más apta para ejercer sus funciones, siempre informada de las innovaciones científicas"; "abre nuevos campos e caminos"; promueve el "reconocimiento de la profesión como parte integrante, necesaria e indispensable para el cuidado a los pacientes, además de crecimiento de los profesionales del área"; favorece una "actuación más profesional de la enfermería y contribuye a establecer la diferencia del área de enfermería relativo a otras profesiones"; auxilia en el "crecimiento personal y en favor del paciente, progreso e incentivo (...)"; lleva la categoría de enfermeras a "participar, contribuir a la evolución profesional en los intercambios de experiencias con éxito, para mejorar la asistencia a nuestro paciente".

Los aspectos que más llaman la atención, por lo reiterado de éstos, son el crecimiento y desarrollo profesional y personal, así como la mejoría de la asistencia al paciente. Vemos, así, que las enfermeras consideran la investigación como un instrumento importante para el crecimiento de la profesión y que, a través de ésta, se puede prestar una asistencia de mejor calidad. Resulta interesante resaltar la preocupación existente con la cuestión asistencial. Aun ocupando cargos de dirección en las unidades en que laboran, estas enfermeras parecen considerar que el bienestar del paciente es el fin último de la asistencia de enfermería. Esto, a nuestro parecer, puede ser consecuencia del énfasis dado a ese asunto en los cursos de graduación.

¿Será que el interés por la investigación aumentaría, en caso que se diera semejante énfasis a la misma? Queremos dejar claro que no creemos que esto resuelva el problema expuesto en el presente trabajo. El énfasis dado en los cursos de graduación, sería apenas uno de los factores que podría promover la participación de los enfermeros de servicio en la producción científica de enfermería. Ya discutimos que muchos de los obstáculos encontrados son externos a la profesión, más no podemos dejar de reconocer que los mismos son reflejo de todo un contexto que incluye cuestiones económicas, políticas y sociales.

El crecimiento y desarrollo profesional a los que se referían algunos de los investigados, no devendrán con el trabajo aislado de uno u otro que confía en su fuerza individual. Éstos sólo se alcanzarán con el reconocimiento colectivo de los profesionales como trabajadores de la salud, inmersos en un contexto multiprofesional y complejo, en que resultan diversos los instrumentos y medios de actuación. La investigación científica es apenas uno de ellos.

\section{CONSIDERACIONES FINALES}

Los cambios que ocurren en el mundo, las innovaciones tecnológicas, los intereses económicos y políticos, entre otros, son factores que ejercen su influencia en el sector de la salud en Brasil, generando modificaciones y necesidades 
específicas en cada momento histórico. Como parte integrante de este sector, la enfermería refleja y, muchas veces, reproduce el cuadro existente en la esfera de la salud en general.

A pesar de las limitaciones y dificultades enfrentadas por la profesión, es innegable el progreso alcanzado en los últimos 50 años, lo cual en el caso de Brasil, viene ocurriendo de forma un poco más lenta. A medida que los estudiosos del área: despliegan esfuerzos con el objetivo de producir un conjunto propio de conocimientos, el empirismo va dando cabida a la práctica basada en el conocimiento científico. No obstante, el desarrollo de la profesión no puede estar circunscrito a esta dimensión, bajo pena de aumentar aún más las fragmentaciones y dicotomías ya existentes, tanto dentro como fuera de la misma.

Entendiendo la enfermería como parte integrante de un complejo sistema multiprofesional, en una visión abarcadora, es que vemos la investigación como instrumento importante en la busca de soluciones para los problemas que emergen de la práctica profesional, interpretando ésta en sus varias dimensiones (asistencia, enseñanza e investigación).

El presente estudio muestra que la situación de las enfermeras de la institución hospitalaria en que fue realizado el estudio, en lo que respecta a la cuestión de la investigación en enfermería, no difiere de otras realidades del país. El análisis de los datos conduce, además, alas siguientes conclusiones:

- la mayoría de las enfermeras nunca realizó, ni realiza investigación actualmente;

- entre las investigaciones realizadas, la mayoría tuvo nexo con la graduación o postgraduación;

- las enfermeras consideran que la falta de tiempo, de asesoramiento y motivación, la dificultad de financiamiento, la insuficiente preparación, la ausencia de incentivo institucional y la inexperiencia, conforman los principales factores limitantes para su inserción en actividades de investigación;

- la mayoría cultiva el hábito de lectura de naturaleza científica, aún cuando no tenga acceso a tal literatura;

- gran parte de las enfermeras considera que la investigación es fundamental para el crecimiento y desarrollo de la profesión, así como para mejorar la calidad de la asistencia prestada al paciente;

- las condiciones de trabajo imperantes en la institución, aparentemente, no favorecen la elaboración de investigaciones por parte de las enfermeras;

- un gran número de las encuestadas manifiesta el deseo de realizar alguna investigación, aún cuando no todas se sienten capacitadas para tal.

Frente a lo anteriormente expuesto, consideramos necesaria la realización de nuevas investigaciones en la institución de referencia, con el fin de evidenciar, de mejor manera aún, el deseo de investigar expresado por las enfermeras, así como lo que ellas consideran capacidad para tal tarea. Los datos obtenidos no revelan claramente estos aspectos, los cuales consideramos de suma importancia para la 
comprensión de lo expresado y de la práctica de las enfermeras participantes de este estudio.

Otro aspecto que juzgamos necesario destacar, es el relativo a que sea creado un núcleo de investigación en enfermería en la institución, considerando el interés demostrado por la dirección del servicio y por el equipo de enfermeras. Sin embargo, para la viabilidad de éste, según nuestro criterio, se necesita una asesoría para la capacitación de las mismas y para el desarrollo de investigaciones de acuerdo con el interés y necesidades sentidas en la práctica profesional.

\section{CARE NURSES AND NURSING RESEARCH: STUDY OF A HOSPITAL REALITY}

The present study aims at demonstrating the situation of nurses of a particular hospital institution with respect to nursing research, focusing their previous and actual research, the limited and favorable elements to elaborate scientific works and the meaning of research to nursing, according to this study participant's opinion. The data collection were made through questionnaire and semi-structured interview.

UNITERMS: nursing research, care nurses

\section{REFERÊNCIAS BIBLIOGRÁFICAS}

01. CASTELLANOS, B. E. P., SALUM, M. J. L. A relação entre a pesquisa e a prática em enfermagem e no setor saúde: reflexões e experiência de enfermeiros de campo num trabalho de pesquisa participante. In: SEMINÁRIO NACIONAL DE PESQUISA EM ENFERMAGEM, 5., Belo Horizonte, 1988. Anais. Belo Horizonte, Associação Brasileira de Enfermagem - Seção Minas Gerais, 1988. p. 41-52.

02. LOPES, C. M. Subsídios para uma reflexão acerca da aplicação de resultados de pesquisas pelo enfermeiro assistencial. Rev. Bras. Enfermagem, v. 42, n. 1, p. 128-33, 1989.

03. MACHADO, P. de A. A enfermagem e a pesquisa. In: CONGRESSO BRASILEIRO DE ENFERMAGEM, 33., Manaus, 1981. Anais. Manaus, Associação Brasileira de Enfermagem - Seção Amazonas, 1981. p. 113-118.

04. MENDES, I. A. C. et al. Acerca da utilização do método científico nas pesquisas de enfermagem. Rev. Bras. Enfermagem, v. 36, n. 1, p. 13-19, 1983. 
05. NOGUEIRA, M. I. de C. A pesquisa em enfermagem e sua aplicação na comunidade. Rev. Bras. Enfermagem, v. 4, n. 2, p. 49-54, 1984.

06. RIBEIRO, C. de M. A pesquisa e a prática de enfermagem. 10: SEMINÁRIO NACIONAL DE PESQUISA EM ENFERMAGEM, 3., Florianópolis, 1984. Anais. Florianópolis, Associação Brasileira de Enfermagem - Seção Santa Catarina, 1984. p. 8-26.

07. ROZEndo, C. A., AlBuQuerque, M. C. dos S. de. Aspectos da produção científica em enfermagem na Universidade Federal de Alagoas, Maceió, Universidade Federal de Alagoas, 1992, 19 p. (datilografado). 